\title{
The Hans Neurath Award lecture of The Protein Society: Proteins-A testament to physics, chemistry, and evolution
}

\author{
JANET M. THORNTON \\ Biochemistry and Molecular Biology Department, University College, WC1E 6BT London, UK \\ Crystallography Department, Birkbeck College, WC1E 7HX, London, UK
}

(RECEIVED November 8, 2000; ACCEPTED November 8, 2000)

\section{Editor's note:}

The following article by Janet Thornton, a summary of her Neurath Award lecture at the Protein Science Symposium of August 2000, is the first of a series of invited articles that Protein Science will publish on the subject of proteomics and bioinformatics this year. Our sincere thanks to Professor Thornton and congratulations on her well-deserved award.

This review is a summary of the talk given when I received The 2000 Hans Neurath Award, presented by Hans Neurath at the Protein Society in San Diego last July. On this occasion, rather than present a standard research talk, I felt it was perhaps more appropriate to present a personal journey through my research into the fundamental principles that relate protein sequence to structure and function. I became interested in proteins in David Phillips' laboratory when only 20 structures had been determined. At this stage, Hans Neurath had been working on proteins for almost 40 years and made many contributions (Neurath 1999; Travis 2000) to progressing the experimental approaches, which allowed us to study proteins in ways we now take for granted. Over the past 25 years, we have seen the field of structural molecular biology expand rapidly, with over 12,000 protein structures now deposited in the Protein Databank (PDB; see http://www.rcsb.org/pdb or http://msd.ebi.ac.uk/), and structure has become ever more important to all life scientists interested in understanding the molecular basis of biological function. This article presents a personal chronological viewpoint, concentrating on some of those areas in which I was involved. However, in some ways it does mirror the progress of the field from one that was often dominated by the physics and chemistry of these beautiful molecules and the difficulties in determining their three-

Reprint requests to: Dr. Janet M. Thornton, Biochemistry and Molecular Biology Department, University College, Gower Street, London WC1E 6BT; e-mail: thornton@ biochem.ucl.ac.uk; fax: 44-171-380-8499.

Article and publication are at www.proteinscience.org/cgi/doi/10.1110/ ps.90001. dimensional (3-D) structures to a wider perspective of understanding how they have evolved and how they work together to orchestrate the biology of life.

\section{Chirality}

Chirality is apparent in many forms of life, from spiraling sweet peas to delicate seashells.

The first protein structures had revealed that the exclusive use of L-amino acids gives rise to the nonsymmetric Ramachandran plot, right-handed $\alpha$-helices and left-handed twisted $\beta$-sheets. However, in 1977, inspection of protein folds led several groups to realize that chirality was also apparent at the higher levels of protein structure, leading to right-handed $\beta \alpha \beta$ units (Fig. 1A) in parallel sheets (Sternberg and Thornton 1976; Nagano 1977; Richardson 1981). Our more recent automated analysis of chirality confirms the strength of this effect, with $95 \%$ of all $\beta \alpha \beta$ units in 271 proteins adopting a right-handed orientation and only $1 \%$ left-handed (4\% being classed as indeterminate; Slidel and Thornton 1995; Fig. 1B). In turn, chirality at the supersecondary structure level has a major influence on the overall topology of protein structures, generating, for example, right-handed four-helix bundles and left-handed TIM barrels (Fig. 1C). This cascade, from L-amino acids to complete topologies, reflects the fact that during folding, proteins are governed by the fundamental rules of physics and chemistry. This strength of preference is very rare in proteins, with most rules (e.g., secondary structure propensities) being much less deterministic. However, as is often the 
A

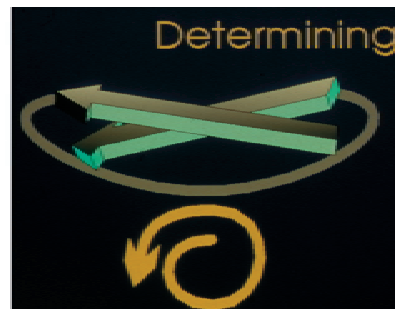

Left-handed

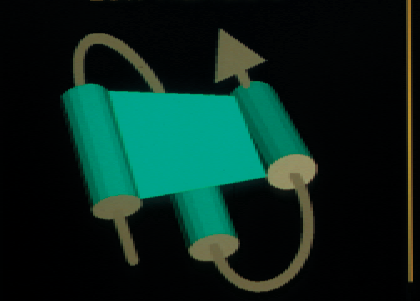

B
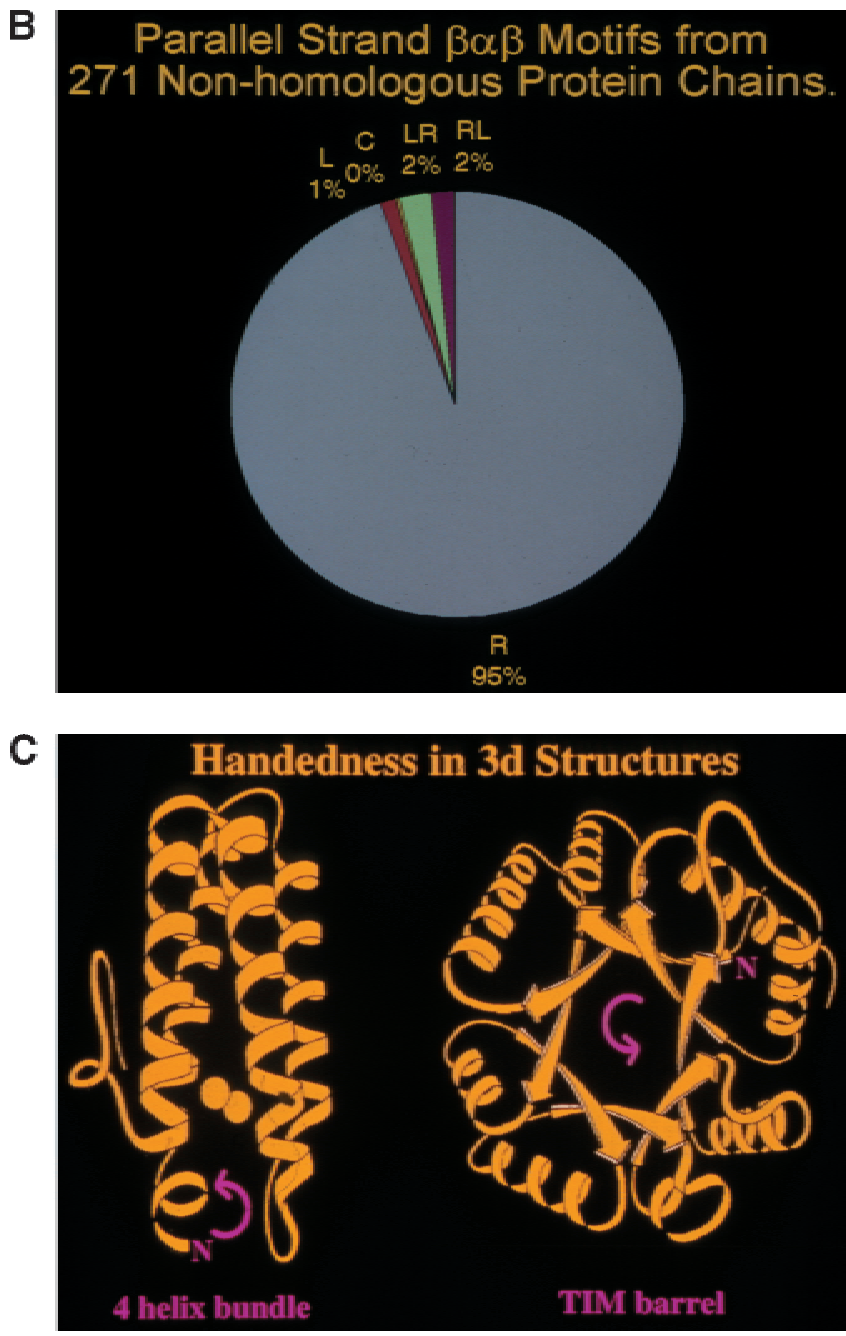

Fig. 1. Chirality in protein structure. (A) Right- and left-handed $\beta \alpha \beta$ units. (B) The distribution of handedness for all $\beta \alpha \beta$-units found in 271 nonhomologous protein structures. (C) Two examples of handedness in domain topology showing the right-handed $4 \alpha$ bundle and the left-handed TIM barrel structure. case, nature surprised us when the structure of UDP-Nacetylglucosamine acetyltransferase (Raetz and Roderick 1995) proved to be a left-handed parallel $\beta$ helix, stabilized by a network of conserved interactions. Even with such strong rules there are exceptions.

\section{Molecular interactions and energetics}

As a postgraduate student, I had worked on dinucleotides, using spectroscopy and semiempirical energy calculations to predict the most stable states of NAD. Even for these small molecules, it was apparent that the potential functions, especially those to model the effects of water, were inadequate to calculate even the enthalpic contribution to free energy. The prospect of tackling proteins and protein folding was therefore daunting. However, as the numbers of protein structures increased, it was clear that we could use these data to begin to understand more about the forces that stabilize proteins. This led to many studies by ourselves and others into specific interactions, for example, $\beta$ turns (Wilmot and Thornton 1988), hydrogen bonds (McDonald and Thornton 1994), disulphides (Thornton 1981), salt bridges (Barlow and Thornton 1983), and side-chain interactions (Singh and Thornton 1990). For example, we found that the different turn types have distinctive amino acid preferences as expected from the Ramachandran plot; disulphides are strongly conserved compared to cysteine residues, reflecting their unique contribution to protein stability and folding; buried salt bridges are very rare, despite having the potential to provide large energies, reflecting the effects of desolvation and evolutionary pressures; and last, that many side chains have preferred packing arrangements, reflecting their inherent chemical natures. As the databases grow, the results of many of these studies are now being refined, but the basic principles were already apparent even with a small database. Such studies have led to an improved qualitative understanding of the energetics of protein structure, although an accurate quantitative approach is still lacking. Much of this physico-chemical information can be captured by deriving empirical potentials from the data (Sippl 1990), though these inevitably incorporate biases inherent in polypeptide chains and so lack the generality expected for a rigorous potential.

\section{Motifs}

One of the ideas that emerged during this time was the concept of supersecondary structures (Rossmann et al. 1974) and motifs, which are common 3-D arrangements of secondary structures that provide the basis for most topologies. For example, $\beta \beta$ hairpins and Greek keys (Richardson 1981) occur frequently and have often been proposed as folding nuclei. 
Although it is now clear that folding is driven more by the burial of hydrophobic groups than the formation of secondary structure units, motifs still provide a useful simplification to describe structures. In addition, they are often found to be the unit of inheritance during evolution, for example, four Greek keys in the $\gamma$-crystallin structure (Blundell et al. 1981). As a corollary to studying the occurrence and structures of $\beta \beta$ hairpins, we analyzed the conformations of loops to explore whether they adopt preferred conformations in these common motifs. We found very specific short loop conformations in most of the common secondary structure motifs (Sibanda and Thornton 1985) that occur in many different unrelated structures and clearly reflect the underlying stability and geometry of these motifs. For example, we found that the unusual type $\mathrm{I}^{\prime}$ and II $^{\prime} \beta$ turns occur almost exclusively in $\beta \beta$ hairpins, where their twist complements that of the $\beta$ strands (see Fig. 2). Such observations highlight the importance of good local packing, which occurs in all structures, despite their tertiary or quaternary complexities. Many such commonly occurring loop motifs have since been recognized and have been successfully incorporated into recent attempts to fold proteins ab initio.

\section{Using stereochemical observations to help detect errors in structures}

Toward the end of the 1980s, several structures were published that were subsequently proven to be incorrect. This led to the call to develop methods that could automatically detect such errors. At about this time, we were also exploring the extent to which the local structure was optimized or compromised by tertiary interactions. For example, how close are $\chi$ angles in folded proteins to their preferred staggered states seen in amino acids in solution? On being asked to present a talk on errors in protein structures at a CCP4 meeting, we began a systematic investigation of the correlation of stereochemical parameters with crystallographic resolution.

Our studies revealed some unusual structures in the PDB that did not seem to agree with expected distributions. Most
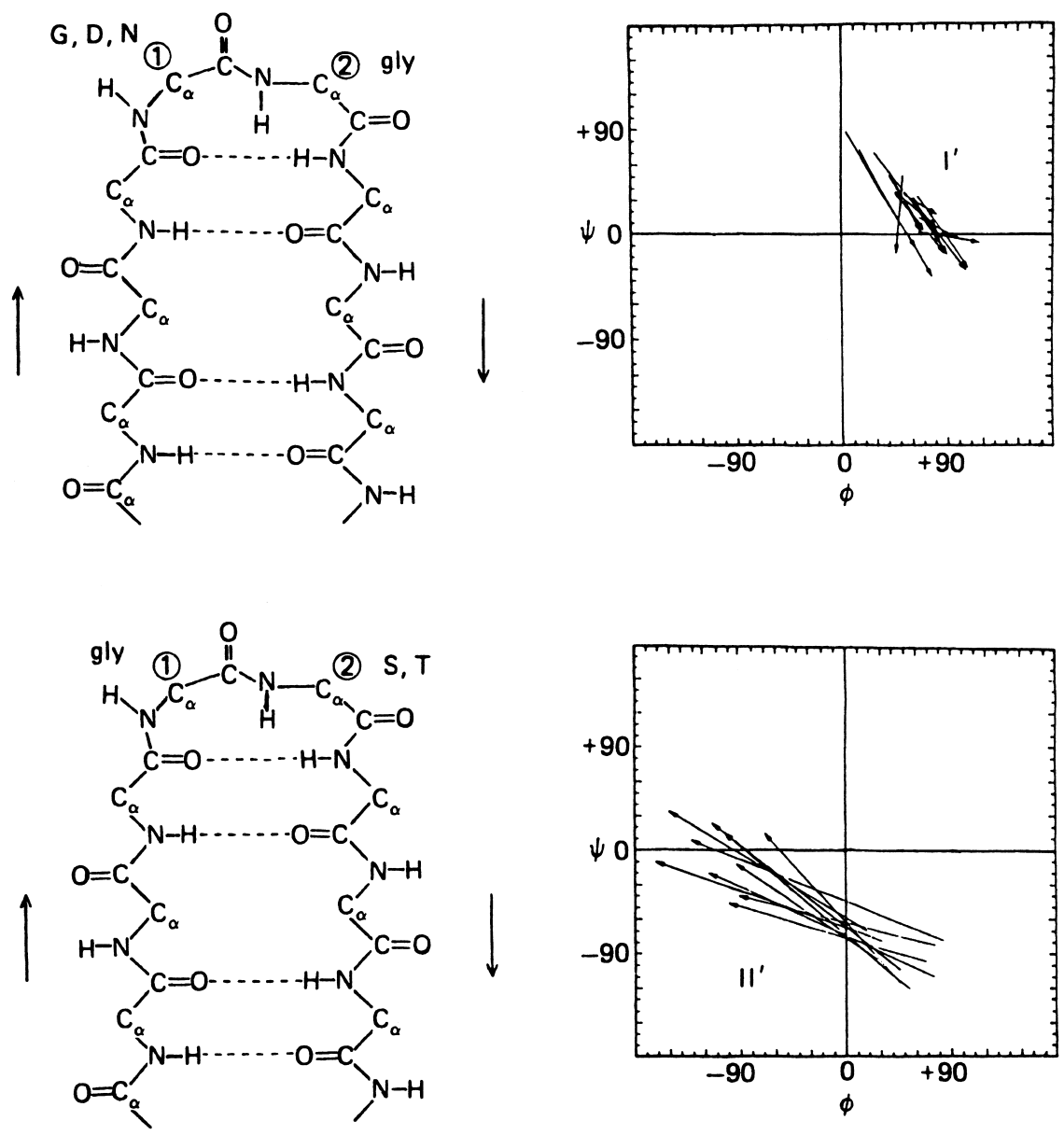

Fig. 2. Two-residue loop motifs in $\beta \beta$ hairpins. For each hairpin, a schematic plot shows the hydrogen-bonding pattern and indicates preferred amino acids for the loop residues. The $\phi \psi$ plots show the conformations of residues 1 and 2 in the hairpin loops, represented by the arrows, with the tail of the arrow representing residue 1 and the head residue 2 . 
parameters we analyzed showed a strong correlation with resolution, excluding those fixed during refinement, which cannot be used as guides to the quality of the structure (Morris et al. 1992). More recent data, extracted from structures determined to atomic resolutions (Butterworth et al. 1998), highlight the remarkably tight distributions seen for many stereochemical parameters (see Fig. 3). Clearly, the conformation of the polypeptide backbone and side chains is close to the minimum energy configuration and is not significantly sacrificed during folding to the native state. These observations provided the basis for the suite of programs PROCHECK (Laskowski et al. 1993), which is now widely used to assess the stereochemical quality of structures. This powerful approach reflects only the physics and chemistry of the structures, with no reference to their biology or evolution.

\section{Classification of protein families by structure-CATH}

As more and more structures were determined, it became clear that it might be possible to provide a classification scheme, analogous to that used for enzymes, to allow protein domains to be grouped into families according to their structures. Such an approach was needed for many reasons, some scientific and fundamental and some technical. To help understand the relationship between sequence and structure and how proteins evolve, we needed the proteins grouped into homologous families according to their evolutionary history.

As some folds are very similar, even when there is no sequence or functional evidence for an evolutionary relationship, we further clustered protein families if their topologies or folds were the same. To divide the structures at a higher level, we also assigned a class (secondary structure content) and architecture (how the secondary structures pack together regardless of sequence order) to each protein domain. This hierarchical classification CATH (Orengo et al. 1997)_representing class, architecture, topology, and homologous family-is based on sequence and structure comparisons using the SSAP algorithm (Taylor and Orengo 1989) combined with manual checking of the results. In the current version of CATH v1.7 (Pearl et al. 2001), there are almost 26,000 domains clustered into 1400 homologous

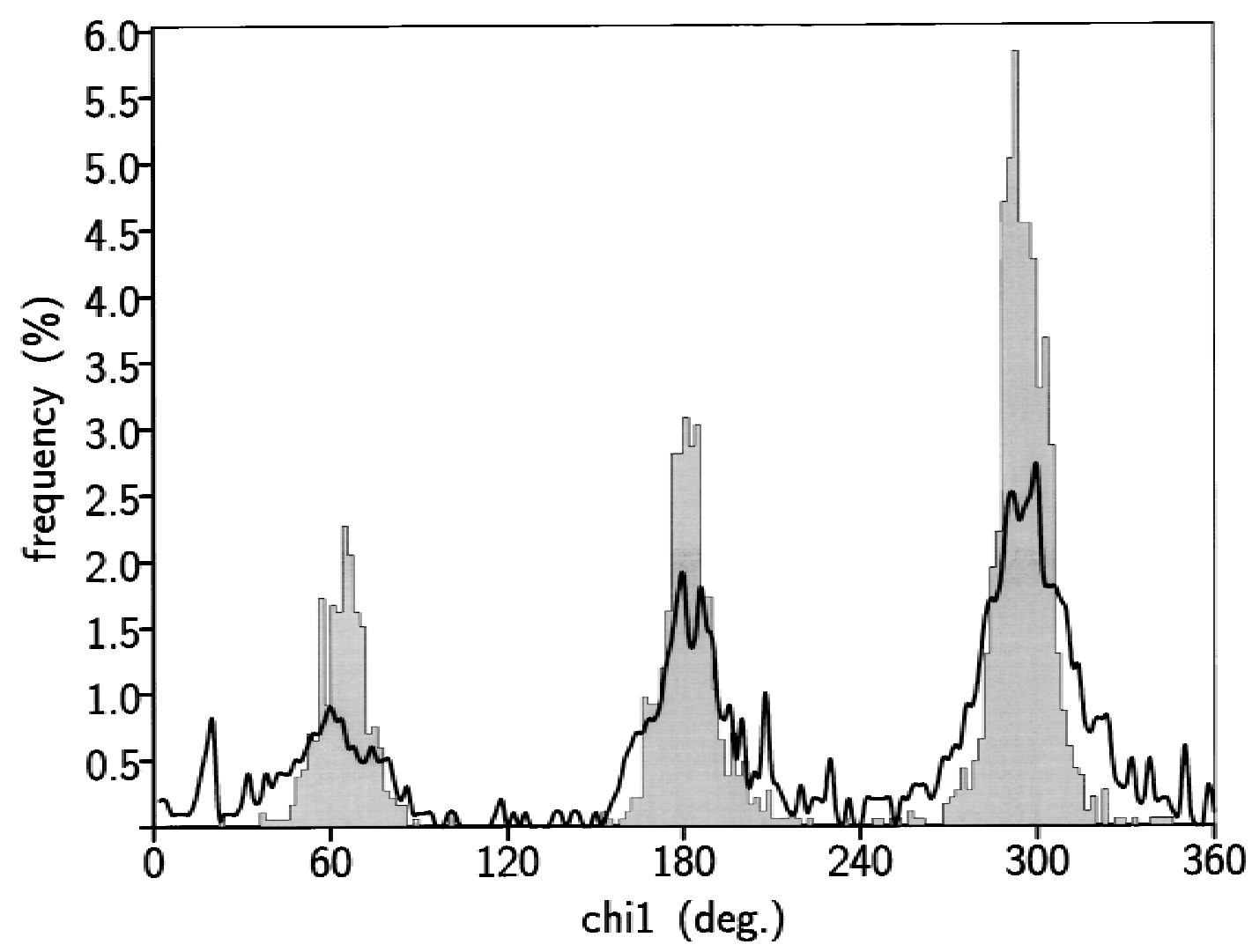

Fig. 3. Comparison of the distribution of $\chi_{1}$ angles in atomic resolution structures (Butterworth et al. 1998) compared to that derived from a set of low-resolution structures extracted from the PDB. From a dataset of 32 crystal structures at atomic resolution, 1851 angles were derived. Superimposed is the envelope of the $\chi_{1}$ distribution for 1901 angles from 10 nonhomologous structures at $2.5 \AA$ resolution. 
families and 592 topologies. In the structurally classified families in the current CATH release, $\sim 25 \%$ families are mainly $\alpha, 21 \%$ are mainly $\beta$, and $>45 \%$ are $\alpha \beta$. Very few $(<8 \%)$ domains have low secondary structure content. Some architectures and folds are disproportionately common. Of all homologous families, $60 \%$ adopt one of eight architectures and $30 \%$ of families adopt one of 10 folds (see Fig. 4). This probably reflects a combination of evolution, by which some families diverge beyond the level at which we can recognize members as being related, and physico-chemical effects, where unrelated sequences converge on the same fold. Other classification schemes (SCOP [Murzin et al. 1995] and FSSP [Holm and Sander 1994]) are based on similar principles, with the majority of assignments in agreement. This database is essential for all our research, which requires validated nonhomologous data sets to avoid bias.

\section{From sequence to structure: Optimal sequence threading for fold recognition}

In 1991, the structure of interleukin-1 $\beta$ was determined (Van Oostrum et al. 1991) and, surprisingly, was found to adopt the same fold as soybean trypsin inhibitor (STI; Sweet et al. 1974). As the coordinates of the interleukin were not made available for some time, we tried to build a model of this structure based on the coordinates of STI. However, it soon became obvious that the sequences were so different (only 17 identical residues out of 170) that the first sequence alignment step, which is necessary to build a model, was not possible using available methods. As more and more structures were deposited in the PDB, it became apparent that the number of novel folds determined each year is rather small. Last year, only $5 \%$ of all newly determined structures adopted a novel fold, although $>75 \%$ of the structures could be identified as relatives by sequence analysis alone. This supports the hypothesis (Chothia 1993; Orengo et al. 1994) that there are a limited number of protein folds from which all the proteins we observe today have evolved. Therefore, we decided to see if we could develop a method to recognize the structure of a new protein by comparison with the available folds in the PDB. Such a fold recognition approach requires three components to solve it: a library of folds, a method to optimally align the sequence onto the structure, and a function to score the match of the sequence with the structure. The novel idea was to calculate the energy in 3-D by building the new sequence into the structure, to use double dynamic programming to find the optimal alignment (Taylor and Orengo 1989), and to score it using Sippl-like (Sippl 1990) potentials. We coined the term threading to describe this approach (Jones et al. 1992). The THREADER program proved very successful for recognizing distant homologs, which cannot be recognized using sequence information alone, but the problem of recog- nizing proteins that adopt similar folds but show no vestige of sequence or functional similarity remains a difficult challenge.

\section{Molecular recognition}

As more structures were determined, the number of protein complexes increased and it became possible to study intermolecular interactions using the PDB. Analyses of proteinnucleotide (Moodie et al. 1996), protein-protein (Jones and Thornton 1996), protein-carbohydrate (Taroni et al. 2000), protein-DNA (Jones et al. 1999), and protein-haem (Karmirantzou and Thornton 1998) interactions in each case reveal that there are many solutions to creating a binding site. For example, a comparison of the adenylate binding sites in unrelated proteins revealed enormous variation in their detailed geometry and residue composition. Only physico-chemical properties, especially hydrophobicity and polarity, at specific atomic sites in the binding pocket are conserved, but the residues providing these atoms are rarely unique. Although this gives rise to distinct residue propensities in binding sites (e.g., aromatics are often involved in sugar binding), their exact location and orientation are highly variable. The concept of a fuzzy recognition template to define a binding site, similar to the pharmacophore model of small molecule ligands, with a few very critical conserved atomic sites but otherwise defined by fuzzy preferences, is a good model. Such observations have helped to provide a foundation to progress rational drug design. The empirical potentials derived from such complexes can be used to rank putative ligands for lead discovery (Mitchell et al. 1999) or protein partners in protein-protein interactions (Ponstingl et al. 2000).

\section{From structure to function}

As more structures become available, we can begin to ask if there is any fundamental relationship between structure and function. Are certain folds more suitable for certain functions as a consequence of their topology, or is the observed mapping from the universe of structures to biological function merely the consequence of evolutionary history? Using the CATH database, we were able to show (Martin et al. 1998) that although there is a slight preference for enzymes to adopt mainly- $\beta$ structures compared to nonenzymes, there is no clear correlation between the Enzyme Commission (E.C.) classification and the protein class. Indeed, the TIM barrel fold performs functions in five of the six primary enzyme classes (Fig. 5). Enzyme functions are determined by a small cluster of catalytic residues that can be placed on almost any framework, although a cleft is usually necessary. In contrast, haem and DNA-binding proteins are dominated by the mainly- $\alpha$ class, presumably because the helices are most suited to creating a hydrophobic cavity for 
A
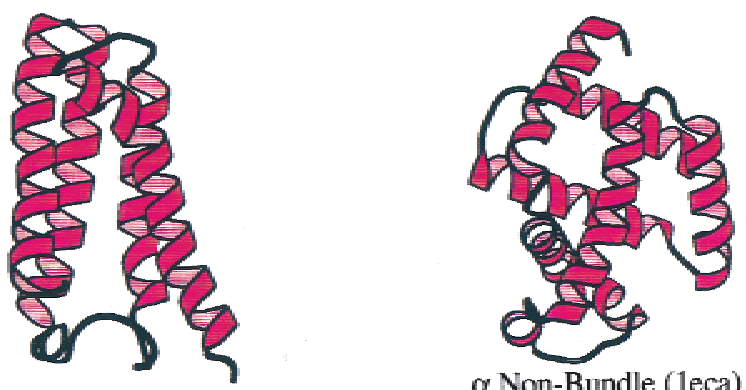

$\alpha$ Non-Bundle (leca)
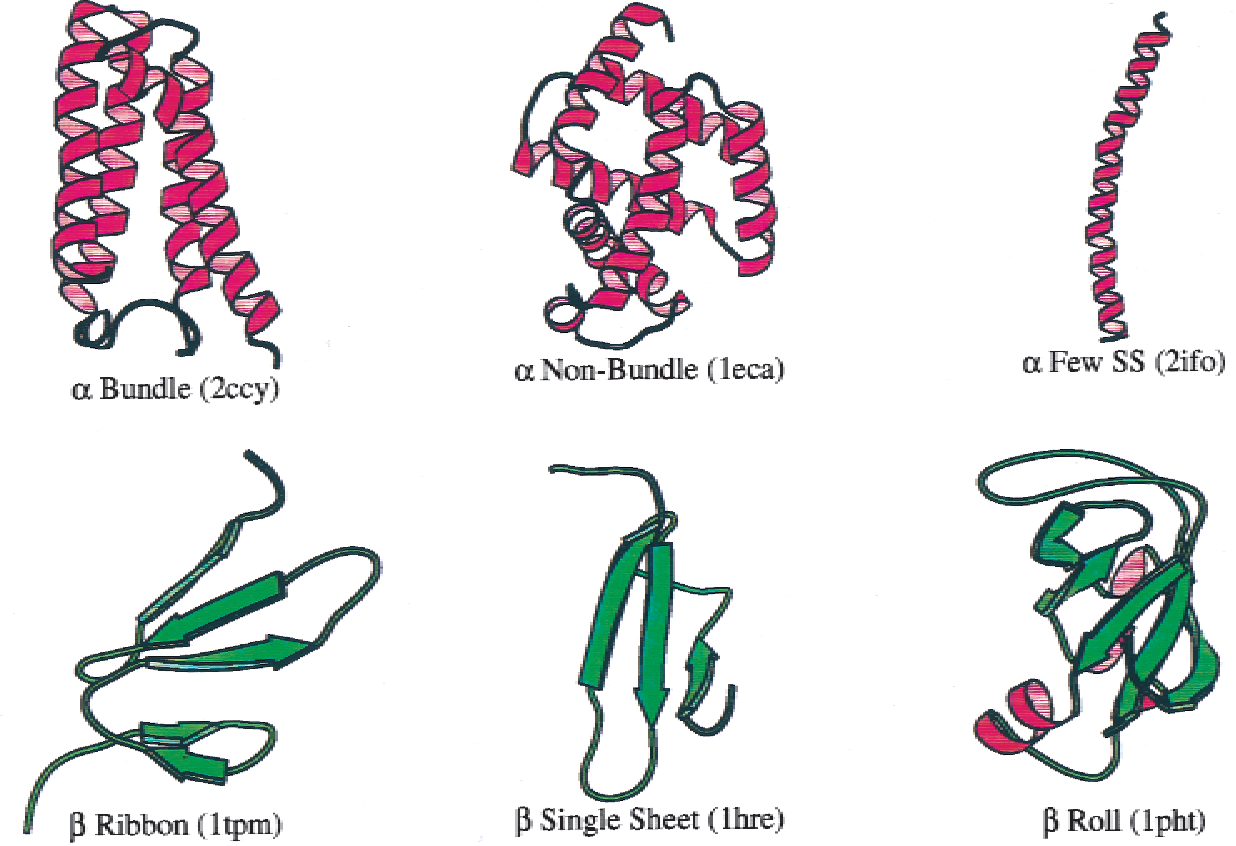

$\beta$ Single Sheet (1hre)

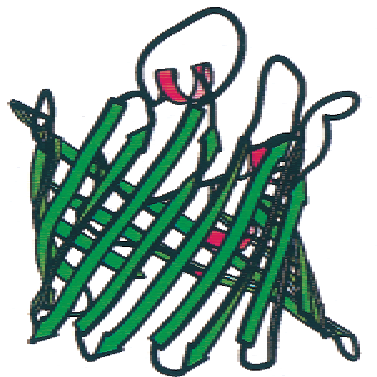

$\beta$ Barrel (2por)
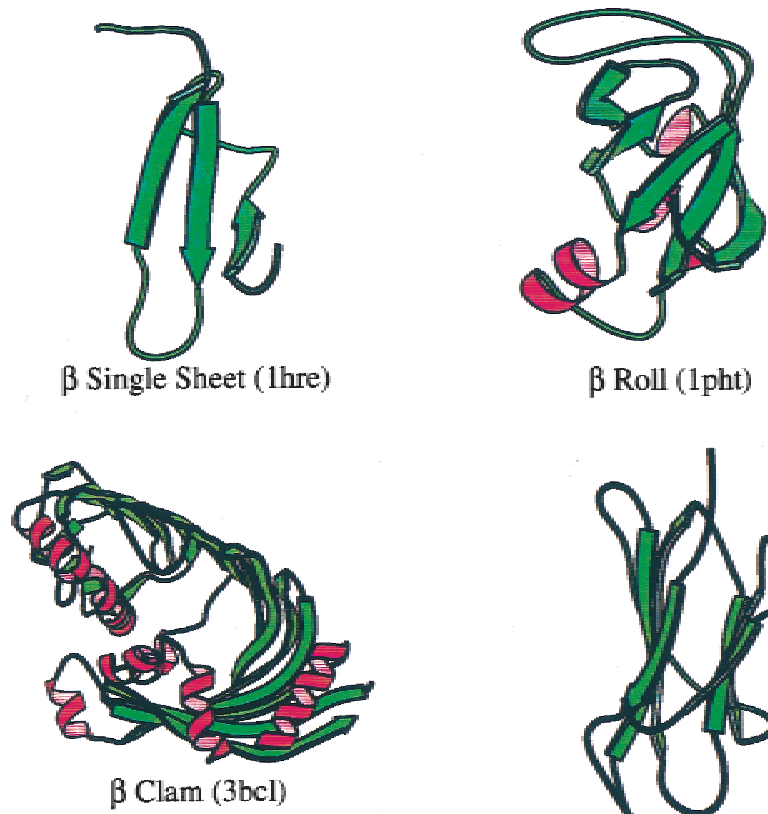

$\beta$ Roll (1pht)
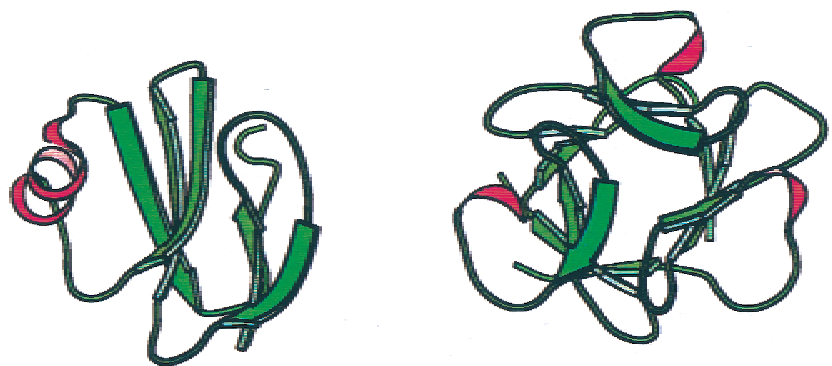

$\beta$ Trefoil (1afcA)

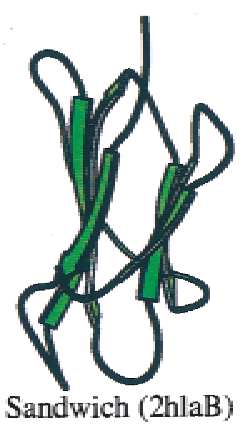

$\beta$ Distorted Sandwich (1cdq)

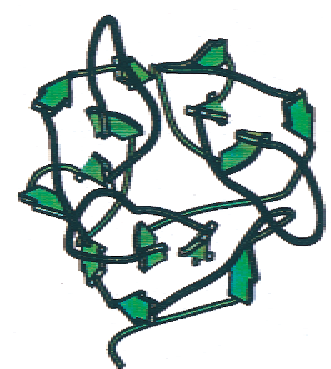

B Aligned Prism (1vmoA)

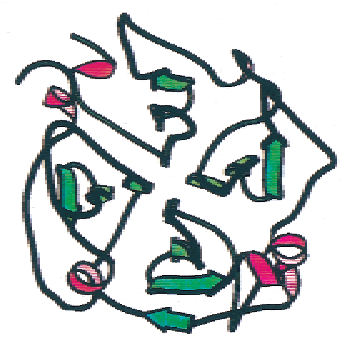

B 4-Propellor (1hxn)

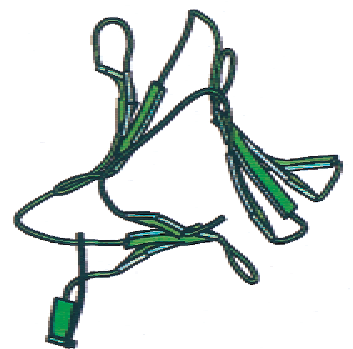

B Orthoganol Prism (lmsaA)

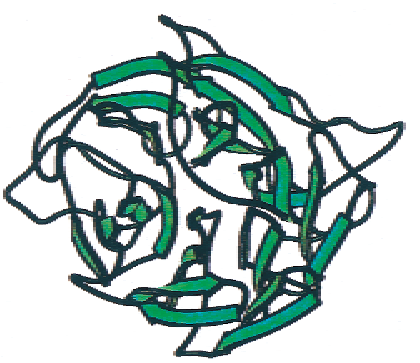

B 6-Propellor (1nscA)

Fig. 4. Structures from the PDB representing the 35 different protein architectures in the CATH protein structure classification system. 
B

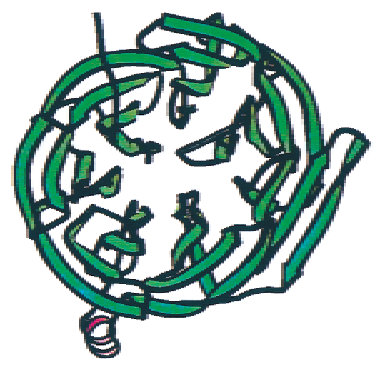

B 7 Propellor (2bbkH)

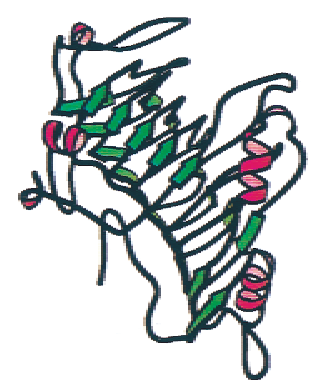

$\beta 3$ Solenoid (2pec)

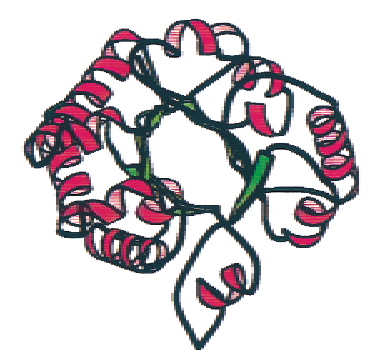

$\alpha \beta$ Barrel (4timA)

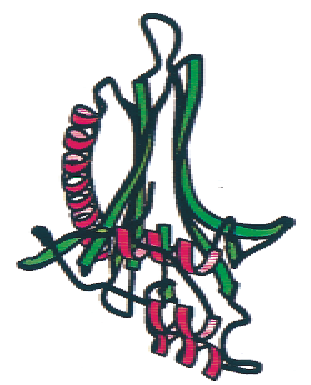

$\alpha \beta$ 3-Layer Sandwich (bba) (1pyaB)

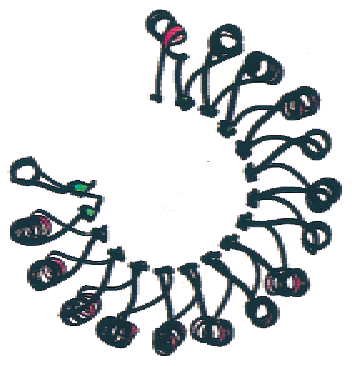

$\alpha \beta$ Horseshoe (lbnh)

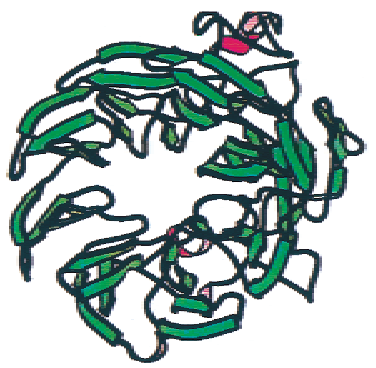

B 8 Propellor (3aahA)

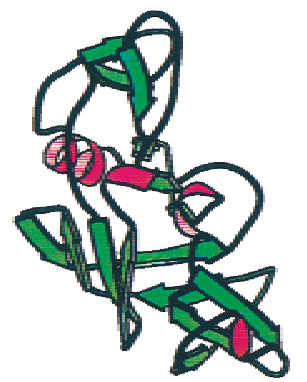

$\beta$ Complex (1ppkE2)

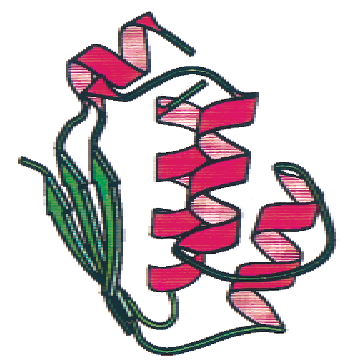

$\alpha \beta$ 2-Layer Sandwich (1brsD)

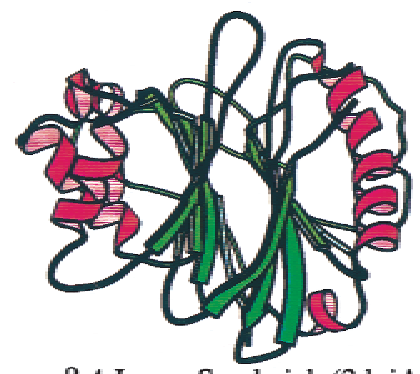

$\alpha \beta$ 4-Layer Sandwich (2dnjA)

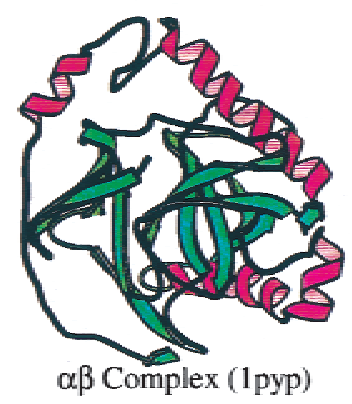

Fig. 4. (Continued.)

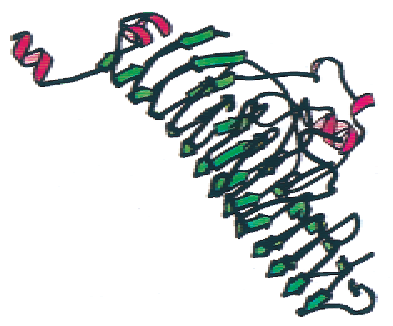

B 2 Solenoid (1tsp)

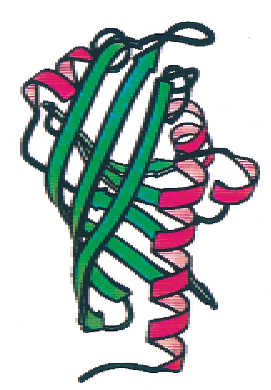

$\alpha \beta$ Roll (1std)

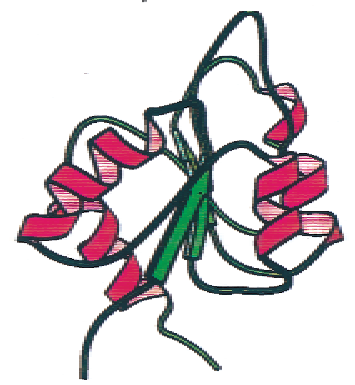

$\alpha \beta$ 3-Layer Sandwich (aba) (1ntr)

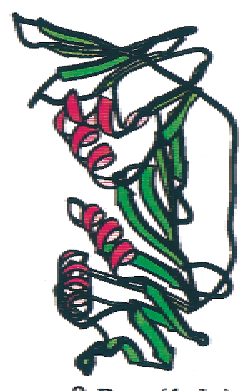

$\alpha \beta$ Box (lplq)

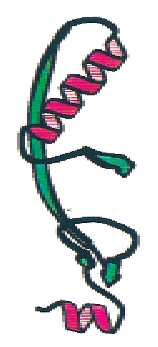

$\alpha \beta$ Few SS (1pyaB) 


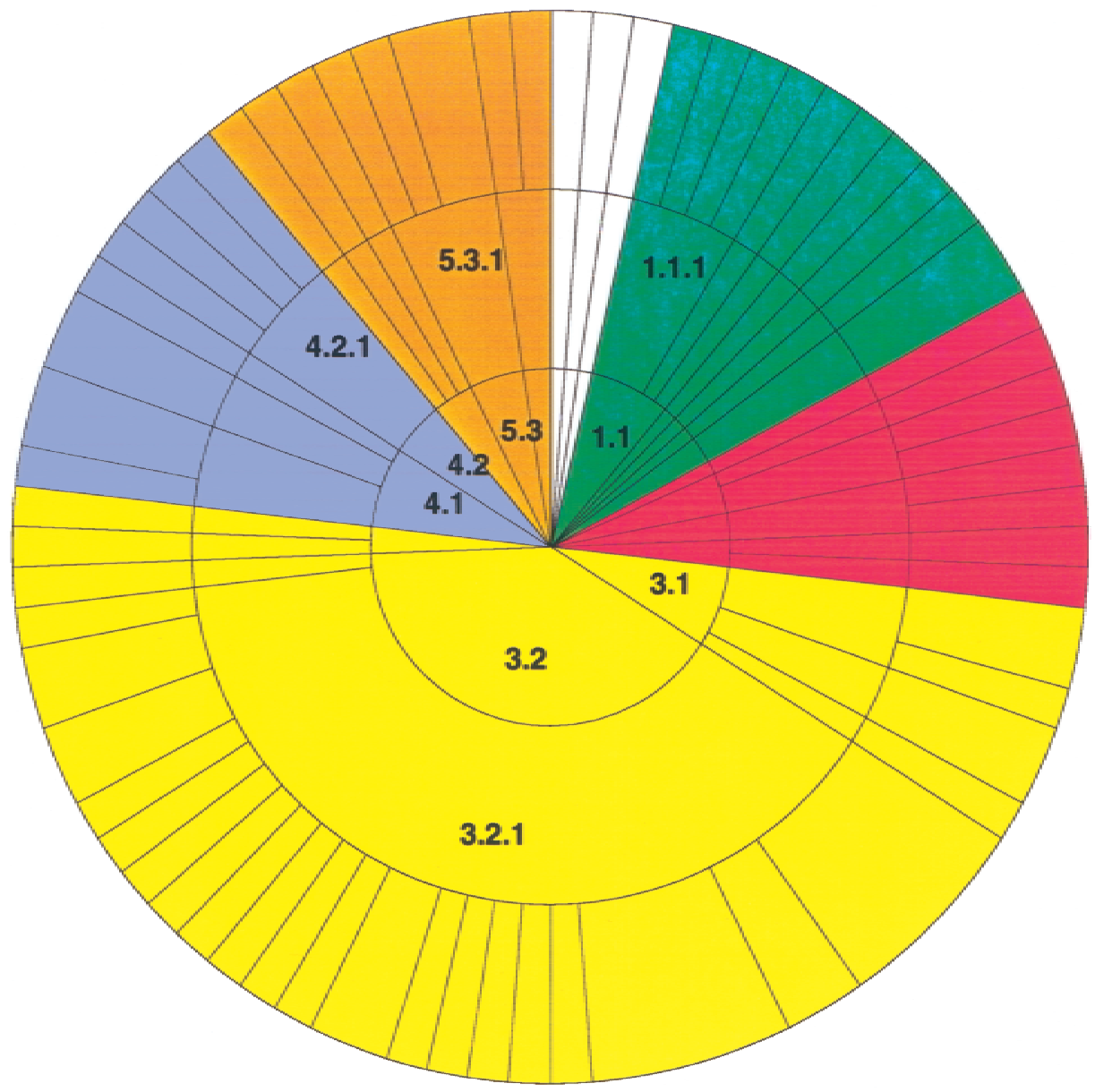

Fig. 5. Functional variation for the TIM barrel $(\beta \alpha)_{8}$ fold. The plot shows the distribution of Enzyme Commission (E.C.) numbers for the 18 homologous families in CATH, which adopt a TIM fold. These proteins are observed to perform the following primary functions: E.C.1, oxidoreductase; E.C.2, transferase; E.C.3, hydrolase; E.C.4, lyase; and E.C. 5, isomerase activities. The primary E.C. numbers are represented by colors, with lower functional descriptors indicated as segments of the wheel.

haem or binding into the major groove of DNA. The nucleotide binding domains are nearly all $\alpha \beta$ proteins, which probably reflects a combination of chemistry, physics, and evolution.

Until the late 1980s, most of the emphasis in the field was on understanding the relationship between sequence and structure, trying to predict structure from sequence, and exploring molecular simulations to analyze the role of conformational change during function. With the realization that structure is much better conserved than sequence, the structural data have become central to understanding the evolution of protein families. Since the pioneering work of Chothia and Lesk (1986), it is clear that the core of the protein structure is conserved, whilst insertions and deletions change the exterior increasingly as the proteins diverge. The extent to which proteins can diverge remains an unanswered question, but it is becoming increasingly clear that they diverge radically in function as well as sequence and structure. In a recent analysis of enzymes in the PDB (Todd et al.
1999), we found $>30$ families with divergent biochemical functions, including radically differing substrates, changes in chemistry, and 20 families that include both enzymes and nonenzymes. These changes usually come about as a result of local mutations, changes in domain composition, or differing quaternary organizations but may also just reflect gene recruitment to a different job in a different environment. Clearly functional annotation of genome sequences will require a more detailed analysis than just assigning a sequence to a given protein family. For this we need to use the data derived from structure to provide residue-based annotation to help discriminate between members of multiple functional families or to help identify distant relatives more reliably.

In recent years, the vast expansion of the field, now known as bioinformatics, has changed what was a rather small esoteric area of biology into mainstream science with important applications in medicine and agriculture. To date, protein structures have been determined mainly to explain 
the known biological function of the protein. The advent of structural genomics and the development of new highthroughput approaches to structure determination will surely lead to a flood of new structures, some without an obvious function. Therefore, the challenge now moves from using the structure to understand biological mechanisms, which has not proved easy, to the even more difficult problem of using the structure to predict molecular interactions and complex biological function. This will involve identifying putative ligands, including small molecules and other proteins, and understanding much more about binding and catalysis. Combined with information from transcriptome and proteome analyses, the next few years will clearly reveal the molecular basis of many aspects of biological function. Hopefully it will also lead us toward rational protein design to allow proteins with novel functions to be engineered. What a glorious challenge for the next 20 years.

\section{Acknowledgments}

It is impossible to acknowledge all those to whom I am indebted and to whom this prize should be jointly awarded. With the exception of one paper, all my work has been collaborative and could not have been done without the input of my colleagues. It has been my delight to work alongside many talented students and research fellows, but here I would particularly like to acknowledge those whose scientific and personal influence have been crucial: David Phillips, Tom Blundell, Peter Bayley, Michael Sternberg, Willie Taylor, Lynn Sibanda, Roman Laskowski, Malcolm MacArthur, Gail Hutchinson, Christine Orengo, David Jones, and Mark Swindells. I would also like to take this opportunity to acknowledge the support and tolerance of my family, without which none of it would be worthwhile.

\section{References}

Barlow, D.J. and Thornton, J.M. 1983. Ion-pairs in proteins. J. Mol. Biol. 168: $867-885$

Blundell, T., Lindley, P., Miller, L., Moss, D., Slingsby, C., Tickle, I., Turnell, B., and Wistow, G. 1981. X-Ray analysis of $\gamma$ crystallin II. Nature 289: 771.

Butterworth, S., Dauter, Z., Dodson, E.J., Hooft, R.W.W., Kaptein, R., Lamzin, V.S., Laskowski, R.A., MacArthur, M.W., Murshudov, T.J., Oldfield, T.J., et al. 1998. Who checks the checkers? Four validation tools applied to eight atomic resolution structures. J. Mol. Biol. 276: 417-436.

Chothia, C. 1993. One thousand families for the molecular biologist. Nature 357: 543-544

Chothia, C. and Lesk, A.M. 1986. The relation between divergence of sequence and structure in proteins. EMBO J. 5: 823-826.

Holm, L. and Sander, C. 1994. The FSSP database of structurally aligned protein fold families. Nucl. Acids Res. 22: 3600-3609.

Jones, D.T., Taylor, W.R., and Thornton. J.M. 1992. A new approach to protein fold recognition. Nature 358: 86-89.

Jones, S., Berman, H., and Thornton, J.M. 1999. Protein-DNA interactions: A structural analysis. J. Mol. Biol. 287: 877-896.

Jones, S. and Thornton, J.M. 1996. Principles of protein-protein interactions. Proc. Nat. Acad. Sci. 93: 13-20.

Karmirantzou, M. and Thornton, J.M. 1998. Computational approaches to protein ligand interactions: Protein-heme complexes. Rational molecular design in drug research, Alfred Benzon Symposium 42. 264-279.

Laskowski, R.A., MacArthur, M.W., Moss, D.S., and Thornton, J.M. 1993. PROCHECK: A program to check the stereo-chemical quality of protein structures. J. Appl. Cryst. 26: 283-291.
Martin, A.C.R., Orengo, C.A., Hutchinson, E.G., Jones, S., Karmirantzou, M., Laskowski, R.A., Mitchell, J.B.O., Taroni, C., and Thornton. J.M. 1998. Protein folds and functions. Structure 6: $875-884$.

McDonald, I.K. and Thornton, J.M. 1994. Satisfying hydrogen-bonding potential in proteins. J. Mol. Biol. 238: 777-793.

Mitchell, J.B.O., Laskowski, R.A., Alex, A., Forster, M., and Thornton, J.M. 1999. BLEEP - A potential of mean force describing protein-ligand interactions. II. Calculation of binding energies and comparison with experimental data. J. Comput. Chem. 20: 1177-1185.

Moodie, S.L., Mitchell, J.B.O., and Thornton, J.M. 1996. Protein recognition of adenylate: An example of a fuzzy recognition template. J. Mol. Biol. 263: 486-500.

Morris, A.L., MacArthur, M.W., Hutchinson, E.G., and Thornton, J.M. 1992. Stereochemical quality of protein-structure coordinates. Proteins Struct. Funct. Genet. 12: 345-364.

Murzin, A.G., Brenner, S.E., Hubbard, T., and Chothia, C. 1995. SCOP: A structural classification of protein database for the investigation of sequences and structures. J. Mol. Biol. 247: 536-540.

Nagano K. 1977. Logical analysis of the mechanism of protein folding. IV. Super-secondary structures. J. Mol. Biol. 109: 235-250.

Neurath, H. 1999. Proteolytic enzymes, past and future. Proc. Natl. Acad. Sci. 96: 10962-10963.

Orengo, C.A., Jones, D.T., and Thornton, J.M. 1994. Protein superfamilies and domain superfolds. Nature 372: 631-634.

Orengo, C.A., Michie, A.D., Jones, S., Jones, D.T., Swindells, M.B., and Thornton. J.M. 1997. CATH-A hierarchic classification of protein domain structures. Structure 5: 1093-1108.

Pearl, F.M.G., Martin N., Bray J.E., Buchan D.W.A., Harrison A.P., Lee D., Reeves G.A., Shepherd A.J., Sillitoe I., Todd A.E., et al. 2001. Nucleic Acids Res. (in press).

Ponstingl, H., Henrick, K., and Thornton, J.M. 2000. Discriminating between homodimeric and monomeric proteins in the crystalline state. Proteins Struct. Funct. Genet. 41: 47-57.

Raetz, C.R. and Roderick, S.L. 1995. A left-handed parallel $\beta$ helix in the structure of UDP-N-acetylglucosamine acyltransferase. Science 270: $997-$ 1000

Richardson, J.S. 1981. The anatomy and taxonomy of protein structure. Adv. Prot. Chem. 34: 167-339.

Rossmann, M.G., Moras, D., and Olsen, K.W. 1974. Chemical and biological evolution of a nucleotide binding protein. Nature 250: 194.

Sternberg, M.J.E. and Thornton, J.M. 1976. On the conformation of proteins: The handedness of the connection between parallel b-Strands. J. Mol. Biol. 110: $269-283$.

Sibanda, B.L. and Thornton, J.M. 1985. $\beta$-Hairpin families in globular-proteins. Nature 316: $170-174$

Singh, J. and Thornton, J.M. 1990. Sirius-An automated-method for the analysis of the preferred packing arrangements between protein groups. J. Mol. Biol. 211: 595-615.

Sippl, M.J. 1990. Calculation of conformational ensembles from potentials of mean force: An approach to the knowledge-based prediction of local structures in globular proteins. J. Mol. Biol. 213: 859-883.

Slidel, T.W.F., and Thornton, J.M. 1995. Chirality in protein structure. In Protein folds: A distance based approach (ed. H. Bohr and S. Brunak), pp. 253-264. CRC, Boca Raton, FL.

Travis, J. 2000. Biography Hans Neurath. Biochim. Biophys. Acta 1477: 3-6.

Sweet, R.M., Wright, H.T., Janin, J., Chothia, C.H., and Blow, D.M. 1974. Crystal structure of the complex of porcine trypsin with soybean trypsin inhibitor (Kunitz) at 2.6- $\AA$ resolution. Biochemistry 13: 4212.

Taylor, W.R. and Orengo, C.A. 1989. Protein structure alignment. J. Mol. Biol. 208: $1-22$.

Taroni, C., Jones, S., and Thornton, J.M. 2000. Analysis and prediction of carbohydrate binding sites. Prot. Eng. 13: 89-98.

Thornton, J.M. 1981. Disulphide bridges in globular proteins. J. Mol. Biol. 151: $261-287$.

Todd, A.E., Orengo, C.A., and Thornton, J.M. 1999. Evolution of protein function, from a structural perspective. Curr. Opin. Chem Biol. 3: 548-556.

Van Oostrum, J., Priestle, J.P., Gruetter, M.G., and Schmitz, A. 1991. The structure of murine interleukin- $1 \beta$ at $2.8 \AA$ resolution. J. Struct. Biol. 107: 189-195.

Wilmot, C.M. and Thornton, J.M. 1988. Analysis and prediction of the different types of $\beta$-turn in proteins. J. Mol. Biol. 203: 221-232. 